\title{
Necrostatin-1 ameliorates the pathogenesis of experimental autoimmune encephalomyelitis by suppressing apoptosis and necroptosis of oligodendrocyte precursor cells
}

\author{
YING WANG, LI GUO, JUEQIONG WANG, WEI SHI, ZHILUN XIA and BIN LI \\ Department of Neurology, The Second Hospital of Hebei Medical University, Shijiazhuang, Hebei 050000, P.R. China
}

Received August 22, 2018; Accepted June 20, 2019

DOI: $10.3892 /$ etm.2019.8005

\begin{abstract}
Multiple sclerosis (MS) is an autoimmune disease of the central nervous system characterized by neuronal demyelination. MS pathogenesis occurs via multiple mechanisms, and is mediated in part by oligodendrocyte apoptosis and a robust inflammatory response. In the present study, Necrostatin-1 (Nec-1), a specific inhibitor of the receptor-interacting protein 1 kinase domain, was revealed to effectively alleviate the severity and pathological damage associated with experimental autoimmune encephalomyelitis (EAE), a commonly used mouse model of MS. In addition, treatment with Nec-1 significantly decreased the number of lesions and inflammatory cell infiltrates in spinal cord tissues, as well as the production of associated pro-inflammatory cytokines, including tumor necrosis factor $\alpha$ (TNF $\alpha)$, interferon- $\gamma$ and interleukin-1 $\beta$. Nec-1 also suppressed $\mathrm{TNF} \alpha+\mathrm{zVAD}$-fmk-induced apoptosis and necroptosis in primary oligodendrocyte precursor cells. The present study revealed that Nec-1 effectively attenuated the progression of EAE by suppressing apoptosis and necroptosis in oligodendrocytes, and represents a potential novel therapeutic agent for the treatment of MS.
\end{abstract}

\section{Introduction}

Multiple sclerosis (MS) is a chronic inflammatory disorder of the central nervous system characterized by lymphocyte, dendritic cell and macrophage infiltration, microglial activation, oligodendrocyte death, demyelination and axonal destruction $(1,2)$. MS is one of the primary causes of neurologic disability in young adults between 20 and 45 years of age (3). The specific pathogenic mechanisms of MS are well

Correspondence to: Dr Bin Li, Department of Neurology, The Second Hospital of Hebei Medical University, 215 Heping West Road, Shijiazhuang, Hebei 050000, P.R. China

E-mail: jack511@163.com

Key words: multiple sclerosis, experimental autoimmune encephalomyelitis, necrostatin-1, oligodendrocytes, apoptosis, necroptosis understood, and a combination of environmental, genetic and infectious factors are implicated in the occurrence and progression of the disease (4). Although the treatment of MS has greatly improved over the past 20 years, further investigation into therapeutic options for this complex disease is required.

Necrostatin-1 (Nec-1) is a potent and specific inhibitor of necroptosis that allosterically suppresses the activity of receptor-interacting serine/threonine protein kinase (RIPK)1, blocking formation of the RIPK1-RIPK3 complex (5). The protective effects of Nec-1 have been reported in a number of experimental models, including models of ischemic brain injury, inflammatory kidney disease, myocardial infarction, Parkinson's disease and various types of cancer (6-11). However, the effects of Nec-1 in MS remain unknown. Therefore, the present study aimed to investigate the functions of Nec-1 in an experimental autoimmune encephalomyelitis (EAE) mouse model.

Necroptosis is a type of programmed cell death with the morphological features of necrosis. Necroptosis is involved in various physiological and pathological conditions, including tissue homeostasis, organ development, ischemia-reperfusion injury, rheumatoid arthritis and neurodegenerative diseases $(12,13)$. It is induced by toll-like receptor and death receptor activation, tumor necrosis factor $\alpha(\mathrm{TNF} \alpha)$, interferons and DNA damage (14), which further activate RIPK3 and its substrate, pseudo-kinase mixed lineage kinase domain-like protein (MLKL) $(15,16)$. Phosphorylated (p)-MLKL is subsequently transformed from the monomeric to the oligomeric form in order to activate downstream signaling cascades and induce programmed cell death (12). Numerous studies have demonstrated the involvement of necroptosis in MS pathogenesis, suggesting that the inhibition of RIPK1 may be an effective means of treating MS $(17,18)$.

$\mathrm{TNF} \alpha$ is an immunomodulatory cytokine that regulates various physiological and pathological functions, including apoptosis, proliferation, inflammation and cancer (19-22). TNF $\alpha$ expression levels are elevated in the serum of patients with MS, which correlates with disease severity (23-26). $\mathrm{TNF} \alpha$-induced oligodendrocyte death morphologically resembles necrosis, and results in the activation of RIPK1 through TNF receptor 1 signaling $(27,28)$.

In the present study, in vivo and in vitro experiments were performed to investigate the functions of Nec-1 in EAE and 
primary oligodendrocytes. Nec-1 significantly attenuated the pathogenesis of EAE by reducing inflammatory factors and suppressing apoptosis and necroptosis. Furthermore, Nec-1 treatment restricted TNF $\alpha+$ zVAD-fmk-induced apoptosis and necroptosis in oligodendrocyte precursor cells (OPCs).

\section{Materials and methods}

Animal maintenance. A total of 24 eight-week-old female C57BL/6 mice (18-20 g) were purchased from Vital River Laboratory Animal Technology Co., Ltd. The mice were housed under specific pathogen-free conditions (temperature, $24 \pm 2^{\circ} \mathrm{C}$; humidity, $50-60 \%$ ) at the animal facility of the Second Hospital of Hebei Medical University (Shijiazhuang, China), with a 12-h light/dark cycle and free access to a standard rodent diet and water. All animal experiments were approved by the animal ethics committee of Hebei Medical University.

EAE induction. Prior to animal experiments, an anesthesia chamber was charged with $1.5 \%$ isoflurane and $100 \%$ oxygen $(2 \mathrm{l} / \mathrm{min}$ oxygen flow) for $15 \mathrm{~min}$, and then mice were put into the chamber for $30 \mathrm{~min}$ to anaesthetize. Each mouse was subcutaneously immunized in the hindquarters with myelin oligodendrocyte glycoprotein (MOG) $)_{35-55}$ (Lysine Biosystem; $250 \mu \mathrm{g}$ for 4 sites) emulsified in an equivalent volume of Complete Freund's Adjuvant (CFA; $90 \%$ paraffin oil and $10 \%$ mannide monooleate, with $4 \mathrm{mg} / \mathrm{ml}$ Mycobacterium tuberculosis, strain H37Ra). Immunized mice also received two intraperitoneal injections of $500 \mathrm{ng}$ pertussis toxin (Alexis Biochemicals; Enzo Life Sciences, Inc.) at days 0 and 2. On day 2, the EAE model was establised. Normal, untreated mice served as the control group.

Nec-1 treatment in vivo. EAE mice were intrathecally injected with $1.65 \mathrm{mg} / \mathrm{kg} \mathrm{Nec}-1$ (Med Chem Express LLC) from day 2 every 3 days for 15 days. Mice in the control group were treated with saline solution only.

Disease scoring. Mice were weighed and evaluated on a daily basis, using the Weaver score method to assess neurological function as previously described (29). The total score ranged from 0 to 14, with a score of between 0 and 2 for the tail, and between 0 and 3 for each of the 4 limbs. For the tail, scoring was defined as follows: i) 0 -no disease; ii) 1-partial paralysis; and iii) 2-paralysis. The limbs were evaluated separately as follows: i) 0-no disease; ii) 1-altered gait or weakness; iii) 2-paresis; and iv) 3-completely paralyzed. Following experiments, at day 30 , mice were sacrificed by $\mathrm{CO}_{2}$ asphyxiation.

Hematoxylin and eosin $(H \& E)$ staining and histopathological scoring. To evaluate inflammatory infiltration, on day 30 , $5-\mu \mathrm{m}$-thick spinal cord tissues sections were fixed with $4 \%(\mathrm{w} / \mathrm{v})$ paraformaldehyde solution overnight at the room temperature in PBS, embedded in paraffin and stained using a conventional H\&E staining method (30). Briefly, tissues were fixed with $10 \%$ paraformaldehyde overnight at room temperature and embedded in paraffin for $2 \mathrm{~h}$ to prepare the paraffin blocks, which were later sliced into $5-\mu \mathrm{m}$-thick sections. Slides were then stained with H\&E staining kit (cat. no. E607318;
Sangon Biotech Co., Ltd.) for $5-10 \mathrm{~min}$ at $20^{\circ} \mathrm{C}$. Following $\mathrm{H} \& \mathrm{E}$ staining, the samples were evaluated using light microscopy at a magnfication of $x 100$. Histopathological scores were calculated as previously described (18): i) 0 -normal; ii) 1-mild inflammation, lymphocyte infiltrates partially surrounding the meninges and blood vessels; iii) 2-moderate inflammation, 1-10 lymphocyte infiltrates in the spinal cord; iv) 3 -severe inflammation, 11-100 lymphocyte infiltrates in the spinal cord; and v) 4-massive inflammation, $>100$ lymphocyte infiltrates in the spinal cord.

Primary cell isolation and culture. At day 16, OPCs were isolated from the cerebrum of C57BL/6 mouse embryos (E16) as described by Chen et al (31). Cells were cultured at $37^{\circ} \mathrm{C}$ in a $5 \% \mathrm{CO}_{2}$ incubator in DMEM/F12 medium (Thermo Fisher Scientific, Inc.) supplemented with $5 \mathrm{ng} / \mathrm{ml}$ neurotrophin 3, $10 \mathrm{ng} / \mathrm{ml}$ ciliary neurotrophic factor, $20 \mathrm{ng} / \mathrm{ml}$ fibroblast growth factor-basic and $20 \mathrm{ng} / \mathrm{ml}$ platelet derived growth factor-AA (all from R\&D Systems, Inc.). Isolated OPCs were identified by staining with anti-platelet derived growth factor receptor $\alpha$ (cat. no. ab134123) and neural/glial antigen 2 (cat. no. ab129051; both 1:200; Abcam) antibodies, and analyzed by flow cytometry using the BD FACSVia ${ }^{\mathrm{TM}}$ system (BD Biosciences). In addition, the primary OPCs were treated with $40 \mathrm{ng} / \mathrm{ml} \mathrm{TNF} \alpha$ and $10 \mu \mathrm{M}$ pan-caspase inhibitor zVAD-fmk (MedChemExpress LLC) for $6 \mathrm{~h}$ to induce apoptosis and necroptosis. For the Nec-1 treatment groups, 20 or $50 \mu \mathrm{M}$ of Nec-1 were applied into the culture medium at the same time TNF $\alpha$ and zVAD-fmk. All the cells were cultured at $37^{\circ} \mathrm{C}$ in a $5 \% \mathrm{CO}_{2}$ incubator in DMEM/F12 medium.

ELISA. Concentrations of the cytokines TNF $\alpha$ (Mouse TNF $\alpha$ Quantikine ELISA Kit; cat. no. PMTA00B), interferon $\gamma$ (IFN $\gamma$; Mouse IFN- $\gamma$ Quantikine ELISA Kit; cat. no. MIF00) and interleukin-1 $\beta$ (IL1 $\beta$; Mouse IL-1 $\beta / \mathrm{IL}-1 \mathrm{~F} 2$ Quantikine ELISA Kit; cat. no. MLB00C) in tissue lysates were determined using the associated ELISA kits (R\&D Systems, Inc.), according to the manufacturer's protocol.

Flow cytometric analysis of apoptosis and necroptosis. Using the fluorescein isothiocyanate-Annexin V Apoptosis Detection kit I (BD Biosciences) according to the manufacturer's protocol, Annexin V/propidium iodide (PI) staining was performed followed by flow cytometry to determine apoptosis and necroptosis. As described previously, $\mathrm{PI} /$ Annexin $\mathrm{V}^{+}$ staining was defined as early apoptosis, $\mathrm{PI}^{+} / A n n e x i n \mathrm{~V}^{+}$ staining as late apoptosis, and $\mathrm{PI}^{+} / A n n e x i n \mathrm{~V}^{-}$staining as pure necroptosis (32).

Reverse transcription-quantitative polymerase chain reaction ( $R T-q P C R)$. RT-qPCR was performed as previously described (33). Total RNA was extracted from the spinal cord tissue and treated cells using Qiagen's RNeasy kit (Qiagen, Inc.) according to the manufacturer's instructions., and cDNA was synthesized using the RevertAid ${ }^{\mathrm{TM}}$ First Strand cDNA synthesis kit (Thermo Fisher Scientific, Inc.). RT-qPCR was detected by the SYBR method [TB Green ${ }^{\circledR}$ Premix Ex Taq ${ }^{\mathrm{TM}}$ II (Tli RNaseH Plus); Takara Bio, Inc.]. A total of $1 \mu \mathrm{g}$ of total RNA was reversely transcribed using oligo(dT) primer at $42^{\circ} \mathrm{C}$ for $1 \mathrm{~h}$, and $2 \mu \mathrm{l}$ of the reverse transcription reaction mix was 
amplified by PCR with denaturation at $95^{\circ} \mathrm{C}$ for $2 \mathrm{~min}$, and 50 cycles at $95^{\circ} \mathrm{C}$ for $30 \mathrm{sec}, 55^{\circ} \mathrm{C}$ for $30 \mathrm{sec}$, and $72^{\circ} \mathrm{C}$ for $1 \mathrm{~min}$. GAPDH was used as an internal control. The $2^{-\Delta \Delta \mathrm{Cq}}$ method was applied to calculate the relative expression (34). Primers for the apoptosis regulators were as follows: Bax forward, 5'-GGA AGGCCTCCTCTCCTACTTC-3' and reverse, 5'-GAGGAC TCCAGCCACAAAGATG-3'; Bcl2 forward, 5'-TTCGCA GCGATGTCCAGTCAGCT-3' and reverse, 5'-TGAAGAGTT CTTCCACCACCGT-3'; Bcl2 like 11 (Bim) forward, 5'-GAG GCGGAGGATGATCCCG-3' and reverse, 5'-CGAGGAGGC AAGGGAAACA-3' and GAPDH forward, 5'-CTGGGCTAC ACTGAGCACC-3' and reverse, 5'-AAGTGGTCGTTGAGG GCAATG-3'.

Western blot analysis. Western blot analysis was performed as previously described (35). Briefly, the samples were lysed with RIPA Lysis Buffer (cat. no. P0013B; Beyotime Institute of Biotechnology) and the protein concentrations were determined by the BCA Protein Assay Kit (cat. no. P0012S; Beyotime Institute of Biotechnology). For each lane, $25 \mu \mathrm{g}$ sample was loaded into $10 \%$ gels; the proteins were separated by SDS-PAGE and transferred to a PVDF membrane. After the transfer, the membrane was blocked with $5 \%$ milk in PBS at room temperature for $1 \mathrm{~h}$. Then, the membrane was incubated with primary antibodies at $4^{\circ} \mathrm{C}$ overnight. On the second day, the membrane was washed with $1 \mathrm{X}$ PBST three times (5 min each) before being incubated with horseradish peroxidase (HRP)-conjuated secondary antibodies for $1 \mathrm{~h}$ at room temperature. The primary antibodies used were antibodies against Bax (cat. no. ab182734), Bim (cat. no. ab7888), Bcl2 (cat. no. ab692), phosporylated-dynamin related protein 1 (p-DRP1; S637; cat. no. ab193216), p-MLKL (S345; cat. no. ab196436), DRP1 (cat. no. ab184248), MLKL (cat. no. ab184718; all 1:1,000; Abcam) and $\beta$-actin (cat. no. AA128; 1:3,000; Beyotime Institute of Biotechnology). HRP-conjugated goat anti-rabbit (cat. no. ZDR5306) or anti-mouse (cat. no. ZDR5307) secondary antibodies (both 1:5,000; OriGene Technologies, Inc.). BeyoECL Plus kit (cat. no. P0018M; Beyotime Institute of Biotechnology) was used to detect the signal. The results were analyzed by ECL detection system (ChemiScope 6000 Ex; Clinx Science Instruments Co., Ltd.) and analyzed by ImageJ software (version 1.52p; National Institutes of Health).

Immunofluorescence staining and confocal imaging. Immunofluorescence staining and confocal imaging were performed as previously described (36). Briefly, treated cells were seeded into a 24-well plate with a polylysine-pretreated coverslip at a density of $1 \times 10^{4}$ cells per well. After treatment, cells were fixed with $4 \%$ paraformaldehyde in PBS for $20 \mathrm{~min}$ at room temperature. The coverslip was then washed twice with PBS (5 min each), incubated with 5\% BSA in 1X TBST in a humidified chamber at room temperature for $1 \mathrm{~h}$ and then washed three times. The slides were incubated with primary p-MLKL antibodies (cat. no. ab196436; 1:200; Abcam) and $\mathrm{Cy} 3{ }^{\circledR}$-conjugated goat anti-rabbit antibodies (cat. no. ab97075; 1:1,000; Abcam). The slides were then washed three times, mounted directly using fluromount with DAPI (Olink Bioscience) and images were collected at a magnfication of x400 using a charge-coupled-device camera (AxioCam MRm) with AxioVision software (version 3; both Carl Zeiss AG). The immunofluorescence staining was analyzed using ImageJ software (version 1.52p).

Statistical analysis. Flow cytometric data were analyzed using FlowJo software 7.6.1 and other data were analyzed using GraphPad Prism 5 software (GraphPad Software, Inc.). For multiple comparisons, one-way analysis of variance was performed followed by Tukey's test. Data are presented as the mean \pm standard error of the mean. $\mathrm{P}<0.05$ was considered to indicate statistical significance.

\section{Results}

Nec-1 reduces the severity of EAE and associated tissue inflammation. To determine whether Nec-1 treatment is able to reduce the disease severity of MS, EAE was induced in C57BL/6 mice using $\mathrm{MOG}_{35-55} / \mathrm{CFA}$. Nec-1 $(1.65 \mathrm{mg} / \mathrm{kg})$ or saline solution were intrathecally administered every 3 days until the end of the experiment (day 30). Nec-1 treatment significantly reduced the severity of EAE in mice (Fig. 1A-C). Furthermore, histological analyses demonstrated that EAE mice possessed a greater number of CNS lesions and inflammatory cell infiltrates in their tissues compared with the sham group (Fig. 1D). This presented as a higher histopathological score for the spinal cord tissue in the EAE group compared with the sham group (Fig. 1E). However, Nec-1 treatment reduced inflammatory cell infiltration and decreased the histopathological score of the spinal cord tissues compared with that in the EAE group (Fig. 1D and E). Inflammatory factors in the tissue lysates of the mice, namely TNF $\alpha$, IFN $\gamma$ and IL1 $\beta$, were evaluated using ELISA kits. The data demonstrated that TNF $\alpha$, IFN $\gamma$ and IL- $1 \beta$ were significantly upregulated in the spinal cord tissues of mice with EAE compared with the sham group (Fig. 1F-H). By contrast, the levels of these inflammatory factors were significantly reduced in Nec-1-treated EAE mice (Fig. 1F-H). These findings suggest that Nec-1 contributed to the reduction of EAE disease severity.

Nec-1 protects spinal cord tissues by reducing MS-associated apoptosis and necroptosis. To further investigate the protective role of Nec-1, the expression levels of apoptosis and necroptosis-associated genes were detected in the spinal cord tissues of mice with EAE, using RT-qPCR and western blotting. The results revealed that EAE induced the expression of pro-apoptotic genes Bax and Bim, and suppressed the expression of anti-apoptotic gene Bcl2. However, Nec-1 treatment significantly decreased the mRNA and protein expression levels of apoptosis-promoting genes Bax and Bim, and increased those of the anti-apoptosis gene Bcl2 following establishment of the EAE model (Fig. 2A-E). This suggests that Nec-1 reduced EAE-associated apoptosis in spinal cord tissues. In addition, EAE suppressed the ratio of pDRP1/DRP and increased the ratio of $\mathrm{p}-\mathrm{MLKL} / \mathrm{MLKL}$, which suggests that EAE induced cell necroptosis in this model. Administration of Nec-1 markedly reversed the changes in the phosphorylation levels of DRP1 and MLKL that were induced in the EAE group, which indicates that Nec-1 also reduced EAE-induced necroptosis in spinal cord tissues (Fig. 2D-F). 

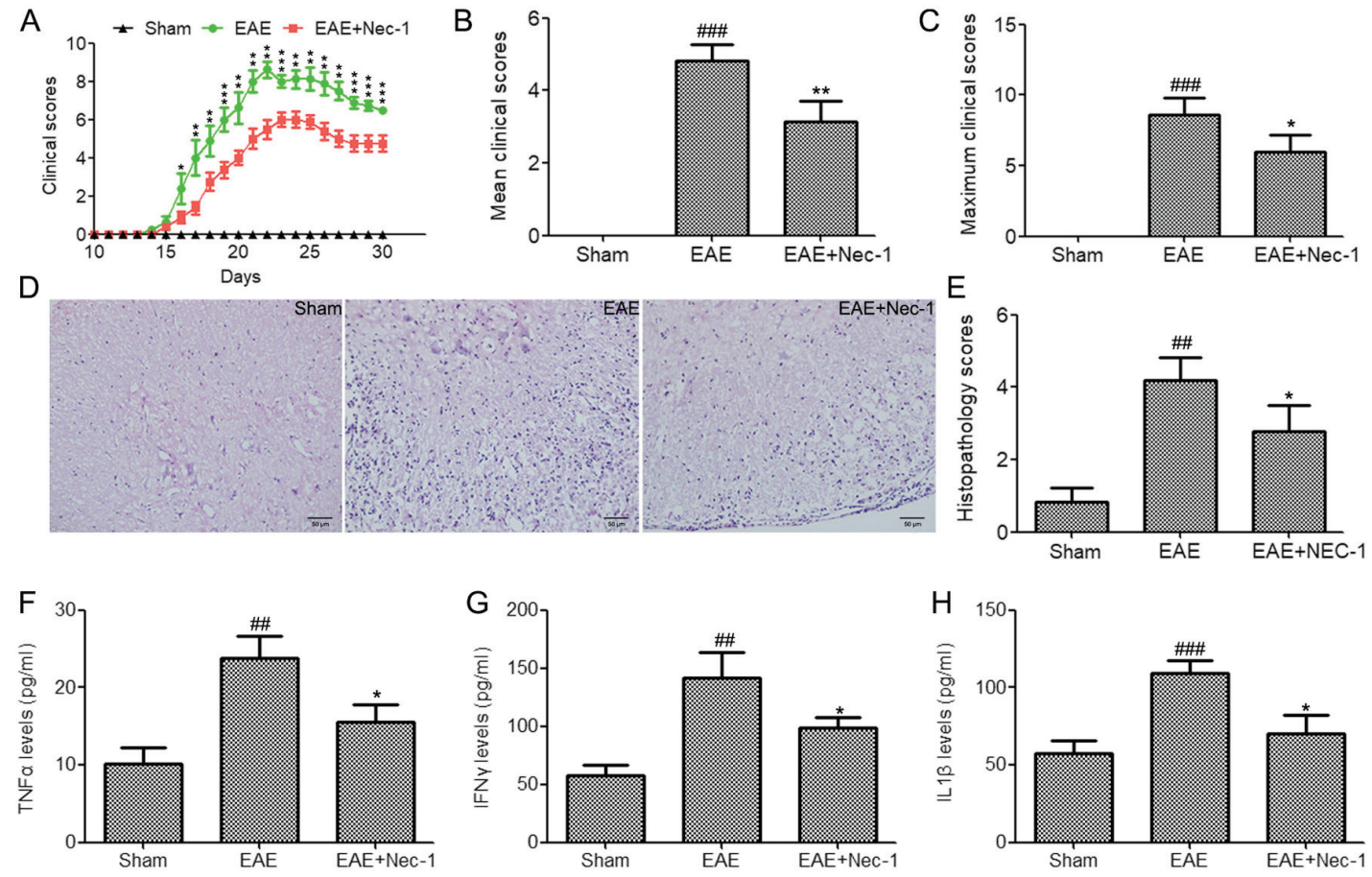

Figure 1. Nec-1 reduces the severity of EAE by suppressing inflammation. (A) Clinical scores of vehicle control and Nec-1 treated mice following immunization with $\mathrm{MOG}_{35-55} /$ Complete Freund's Adjuvant. (B) Mean and (C) maximum clinical scores of indicated animal models. (D) Hematoxylin and eosin staining and (E) histopathology scores of spinal cord tissue sections from the sham, EAE and EAE + Nec-1 groups. (F-H) ELISA was used to determine the expression of (F) TNF $\alpha$, (G) IFN $\gamma$ and (H) IL-1 $\beta$ in spinal cord tissues. $n=8$ animals per group. ${ }^{*} \mathrm{P}<0.05,{ }^{* * *} \mathrm{P}<0.01$ and ${ }^{* * * *} \mathrm{P}<0.001$ vs. EAE; ${ }^{\# \#} \mathrm{P}<0.01$ and ${ }^{\# \# \#} \mathrm{P}<0.001$ vs. sham. Nec-1, Necrostatin-1; EAE, experimental autoimmune encephalomyelitis; TNF $\alpha$, tumor necrosis factor $\alpha$; IFN $\gamma$, interferon $\gamma ;$ IL-1 $\beta$, interleukin- $1 \beta$.
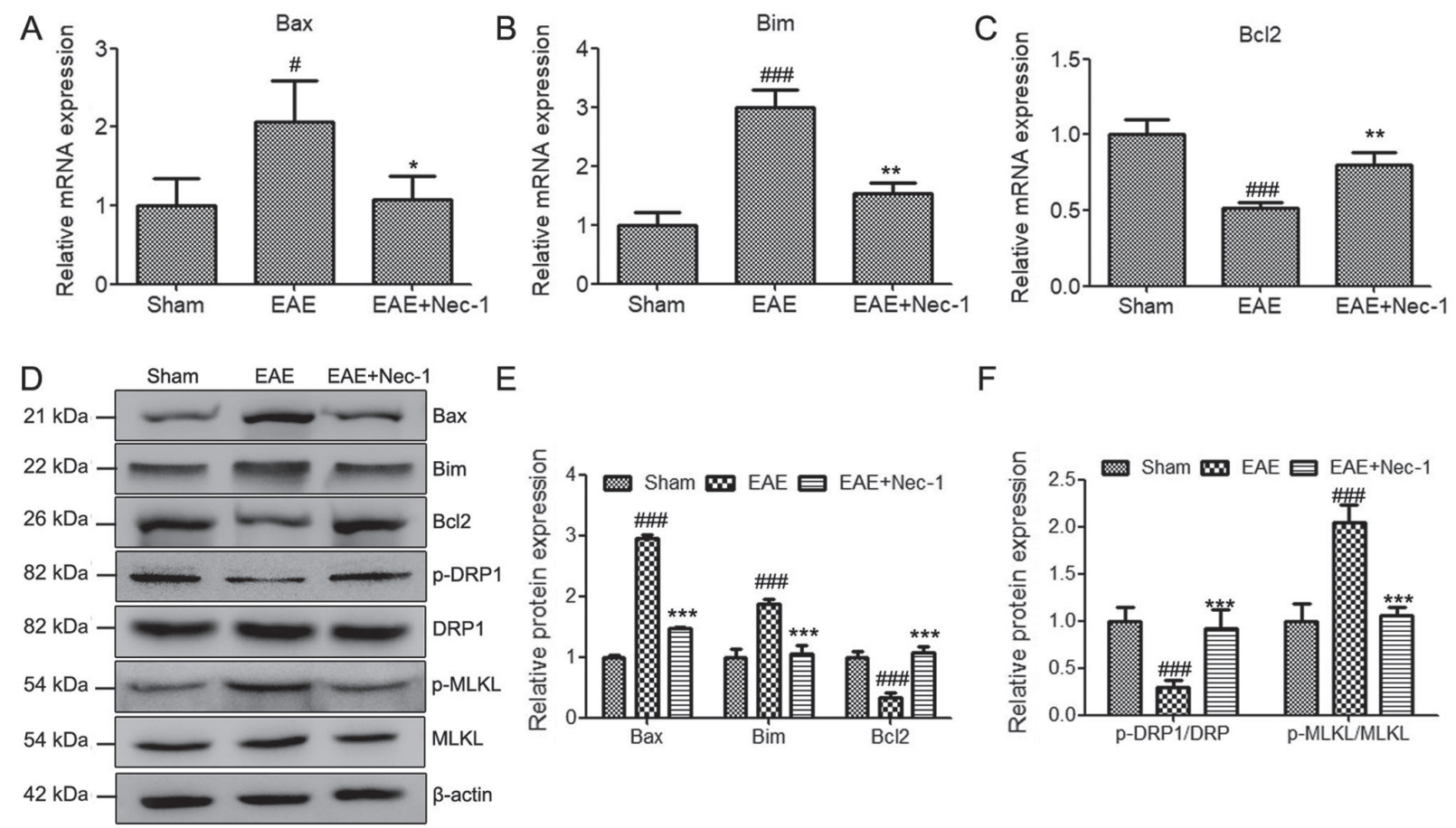

E

$\mathrm{F}$
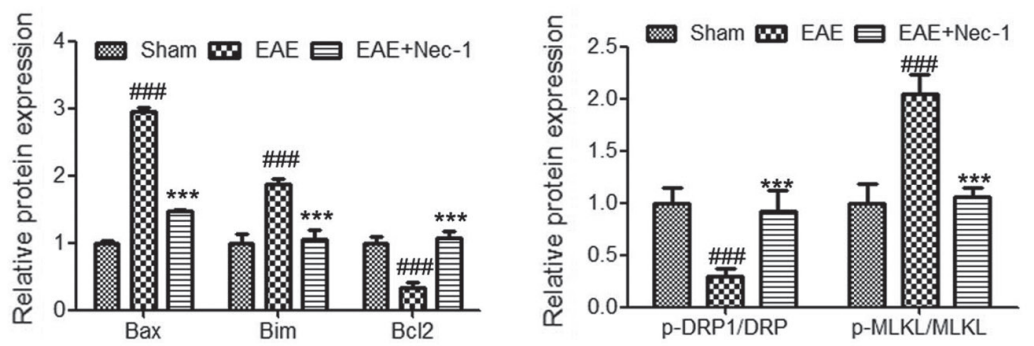

Figure 2. Nec-1 reduces cellular apoptosis and necroptosis in spinal cord tissues. (A-C) Reverse transcription-quantitative polymerase chain reaction was used to determine the expression levels of (A) Bax, (B) Bim and (C) Bcl2 in spinal cord tissues. (D) Representative western blots, (E) quantified protein expression levels of Bax, Bim and Bcl2 and (F) p-DRP1/DRP and p-MLKL/MLKL ratios in spinal cord tissues. " $\mathrm{P}<0.05$ and ${ }^{\# \# \# /} \mathrm{P}<0.001$ vs. sham control; " $\mathrm{P}<0.05$, ${ }^{* *} \mathrm{P}<0.01$ and ${ }^{* * * *} \mathrm{P}<0.001$ vs. EAE. Nec-1, Necrostatin-1; Bim, Bc12 like 11; p-DRP1, phosphorylated-dynamin related protein 1; p-MLKL, phosphorylated pseudo-kinase mixed lineage kinase domain-like protein; EAE, experimental autoimmune encephalomyelitis.

Nec-1 inhibits apoptosis and necroptosis in primary OPCs induced using $T N F \alpha+z V A D-f m k$. It has previously been reported that the apoptosis and necrosis of oligodendrocytes is fundamental to the initiation and progression of EAE (37). 
A

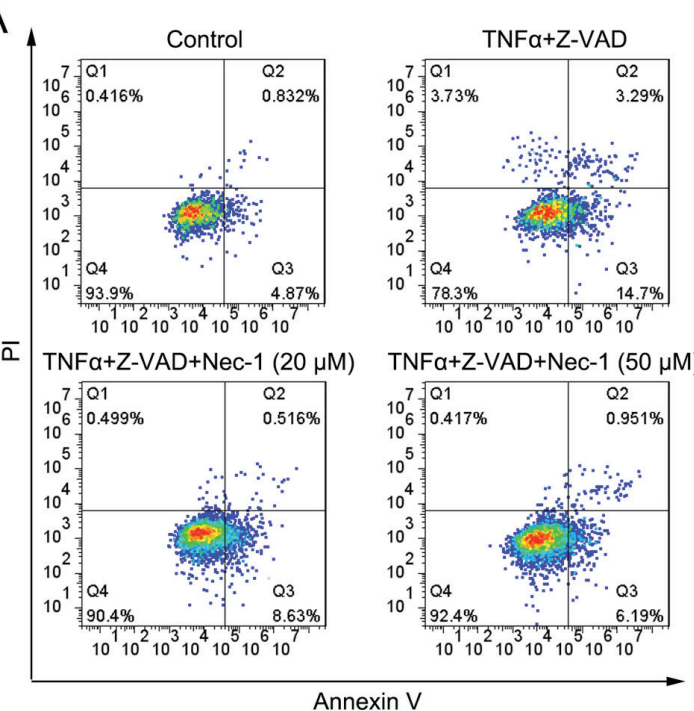

B

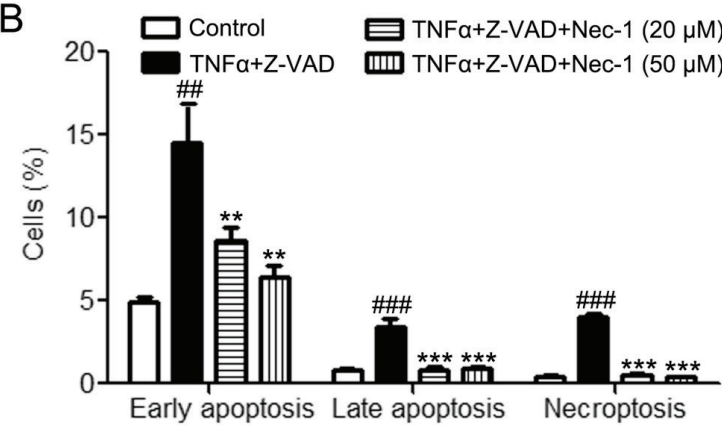

C

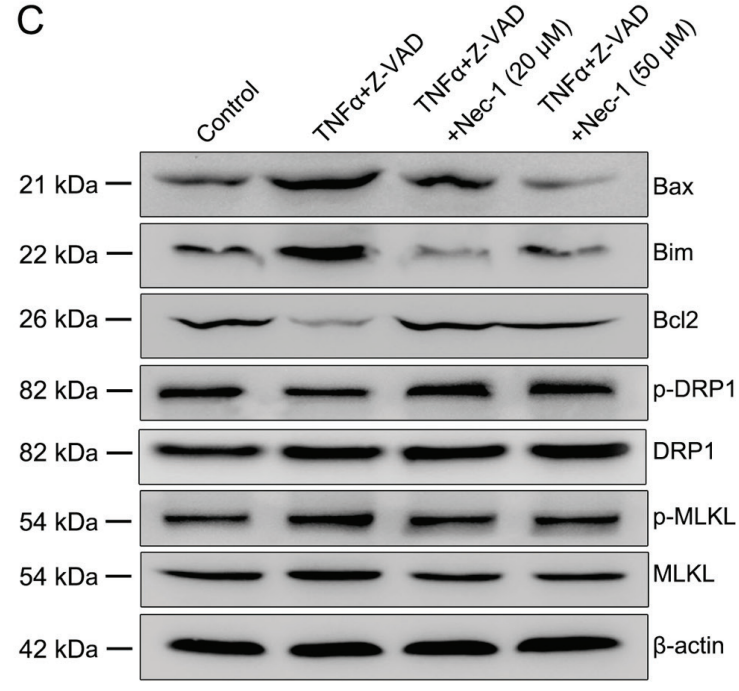

$\mathrm{D}$
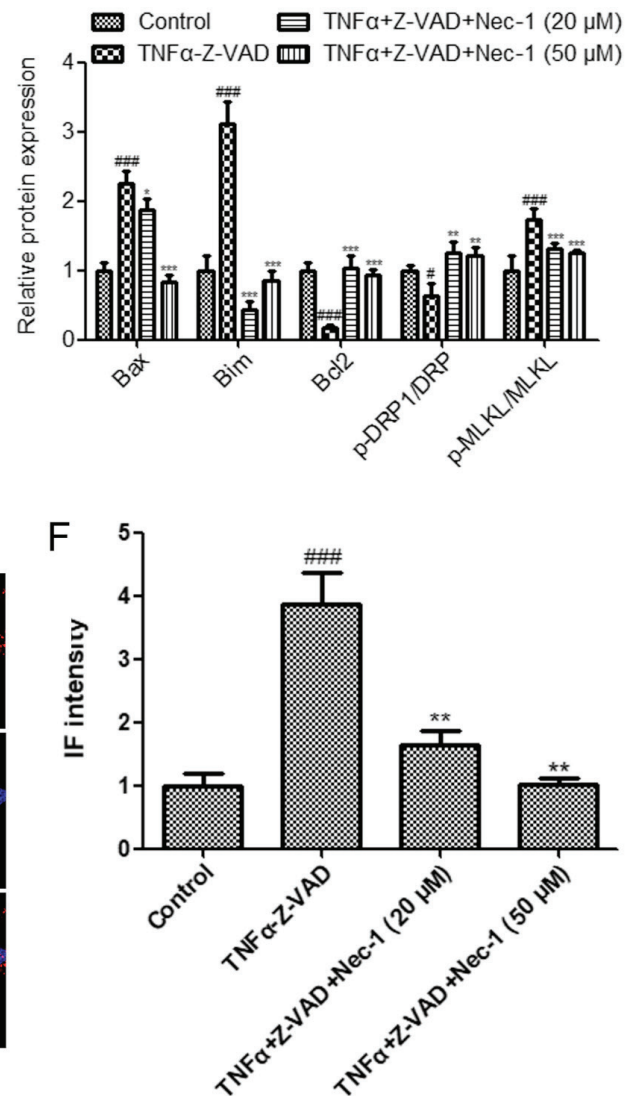

Figure 3. Nec-1 protects mouse OPCs against apoptosis and necroptosis induced by TNF $\alpha+$ zVAD-fmk. (A) Annexin V and PI staining to determine

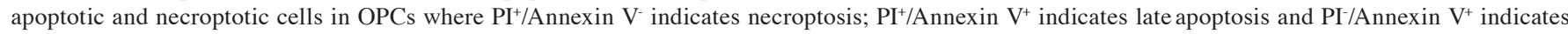
early apoptosis. (B) Percentage distributions of apoptotic and necroptotic cells. (C) Representative western blots and (D) quantification of Bax, Bim and $\mathrm{Bcl} 2$ expression, and p-DRP1/DRP and p-MLKL/MLKL ratios in OPCs with the indicated treatment. (E) Immunofluorescence staining micrographs and (F) quantification of staining intensity to detect the expression and localization of $\mathrm{p}-\mathrm{MLKL}$ in OPCs. ${ }^{*} \mathrm{P}<0.05,{ }^{\# \#} \mathrm{P}<0.01$ and ${ }^{\# \# \#} \mathrm{P}<0.001$ vs. control; ${ }^{* *} \mathrm{P}<0.01$ and ${ }^{* * * *} \mathrm{P}<0.001$ vs. TNF $\alpha+$ zVAD-fmk. Nec-1, Necrostatin-1; OPCs, oligodendrocyte precursor cells; TNF $\alpha$, tumor necrosis factor $\alpha$; PI, propidium iodide; p-DRP1, phosphorylated-dynamin related protein 1; p-MLKL, phosphorylated pseudo-kinase mixed lineage kinase domain-like protein.

Therefore, the protective effect of Nec-1 on oliogodendrocytes in EAE was investigated. Primary OPCs were isolated (Fig. S1) and $\mathrm{TNF} \alpha$ and the pan-caspase inhibitor zVAD-fmk were administered to primary OPCs to induce apoptosis and necroptosis. The results demonstrated that $\mathrm{Nec}-1$ treatment (20 and $50 \mu \mathrm{M})$ significantly reduced the number of necroptotic and apoptotic cells induced by TNF $\alpha+z V A D-f m k$ (Fig. 3A and B). In addition, western blot analyses were performed to evaluate the protein levels of Bax, Bim, Bcl2, p-DRP1 and p-MLKL in these groups. In agreement with the in vivo results, the administration of TNF $\alpha+z V A D-f m k$ significantly upregulated the expression levels of Bax and Bim, and the phosphorylation of 
MLKL, and reduced DRP1 phosphorylation and Bcl2 expression in OPCs (Fig. 3C and D). Nec-1 treatment (20 and $50 \mu \mathrm{M})$ reversed the EAE-induced changes in the levels of these proteins (Fig. 3D). Similar trends were observed for the immunofluorescence staining of p-MLKL in the indicated groups, where TNF $\alpha+$ zVAD-fmk significantly increased p-MLKL staining intensity which was then attenuated by Nec-1 treatment at both concentrations (Fig. 3E and F). These results demonstrated that Nec-1 suppressed the TNF $\alpha+z V A D$-fmk-induced apoptosis and necroptosis of OPCs.

\section{Discussion}

EAE is a widely adopted animal model that mimics the clinical characteristics and pathogenic mechanisms of MS, a disease affecting 2.5 million individuals worldwide (38). The EAE animal model provides a suitable tool for understanding the pathogenesis and underlying mechanisms of MS and also how best to manage the condition (39). Using the EAE model, the present study established that necroptosis inhibitor Nec-1 reduced the pathogenesis of MS by the suppression of apoptosis and necroptosis in OPCs. To the best of our knowledge, this is the first study to directly demonstrate the protective role of Nec-1 in EAE and MS, with the results providing background for the potential discovery of novel therapies.

A large number of studies have demonstrated that necroptosis may contribute to the pathogenesis of various neurodegenerative disorders, including MS $(13,40,41)$. The activation of RIPK1, RIPK3 and MLKL has been observed in cortical lesions from MS brain specimens (17). Ofengeim et al (18) demonstrated that necroptosis is involved in MS, and suggested that targeting RIPK1 may represent a therapeutic strategy for treating the disease. The inhibition of necroptosis may also provide effective relief from the symptoms of MS. Previous studies have illustrated that Nec-1 has a significant neuroprotective effect in ischemic stroke $(6,42,43)$. However, the role of Nec-1 in MS remains unknown. In the present study, the use of in vivo and in vitro models revealed for the first time that Nec-1 effectively alleviates the symptoms of EAE. Mechanistically, Nec-1 reduced cellular apoptosis and necroptosis induced by EAE in vitro and in vivo, providing an improved understanding of the role of Nec-1 in MS.

Microglial cells are resident innate immune cells, primarily accountable for the inflammatory response in neurodegenerative diseases $(43,44)$. Since RIPK1 is more highly expressed than RIPK3 in activated microglia (17), targeting RIPK1 may selectively inhibit microglial-mediated inflammatory signaling. The present study demonstrated that $\mathrm{Nec}-1$ suppressed the expression of inflammatory cytokines TNF $\alpha$, IFN $\gamma$ and IL1 $\beta$ in spinal cord tissues. Microglia-associated inflammation triggers necroptosis in oligodendrocytes, which is critical for the pathogenesis of MS $(17,44)$. Therefore, the present study illustrated that Nec-1 may effectively regulate inflammation in microglial cells, and necroptosis in oligodendrocytes.

Several limitations exist for the present study. For instance, although it was determined that Nec-1 suppressed the apoptosis and necroptosis of primary oligodendrocyte precursor cells in vitro, no histological staining for the apoptosis or necroptosis of oligodendrocytes in the spinal cord tissues in vivo was performed. Future studies will involve more in-depth in vivo experiments.
In conclusion, the present study demonstrated that Nec-1 suppressed the apoptosis and necroptosis of oligodendrocytes by inhibiting the release of inflammatory mediators. The promising therapeutic value of Nec-1 for the treatment of MS was demonstrated, suggesting that it may have potential positive neuroprotective effects on patients with MS.

\section{Acknowledgements}

The authors greatly thank the Neurology Laboratory of The Second Hospital of Hebei Medical University (Shijiazhuang, China).

\section{Funding}

The current study was supported by the National Natural Science Foundation of China (grant no. 81873759).

\section{Availability of data and materials}

All data generated or analyzed during this study are included in this published article.

\section{Authors' contributions}

BL proposed the current study and drafted the manuscript. LG designed the experiments. YW performed the experiments and wrote the manuscript. JW collected and analyzed experimental data. ZX and WS performed histological analysis. All authors read and approved the final manuscript for publication.

\section{Ethics approval and consent to participate}

All animal experiments were approved by the animal ethics committee of Hebei Medical University.

\section{Patient consent for publication}

Not applicable.

\section{Competing interests}

The authors declare that they have no competing interests.

\section{References}

1. Sedal L, Winkel A, Laing J, Law LY and McDonald E: Current concepts in multiple sclerosis therapy. Degener Neurol Neuromuscul Dis 7: 109-125, 2017.

2. Lemus HN, Warrington AE and Rodriguez M: Multiple Sclerosis: Mechanisms of disease and strategies for myelin and axonal repair. Neurol Clin 36: 1-11, 2018.

3. Kacperska MJ, Walenczak J and Tomasik B: Plasmatic microRNA as potential biomarkers of multiple sclerosis: Literature review. Adv Clin Exp Med 25: 775-779, 2016.

4. Simon M, Ipek R, Homola GA, Rovituso DM, Schampel A, Kleinschnitz C and Kuerten S: Anti-CD52 antibody treatment depletes B cell aggregates in the central nervous system in a mouse model of multiple sclerosis. J Neuroinflammation 15: 225, 2018.

5. Linkermann A, Hackl MJ, Kunzendorf U, Walczak H, Krautwald S and Jevnikar AM: Necroptosis in immunity and ischemia-reperfusion injury. Am J Transplant 13: 2797-2804, 2013. 
6. Yang R, Hu K, Chen J, Zhu S, Li L, Lu H, Li P and Dong R: Necrostatin-1 protects hippocampal neurons against ischemia/reperfusion injury via the RIP3/DAXX signaling pathway in rats. Neurosci Lett 651: 207-215, 2017.

7. Tristao VR, Goncalves PF, Dalboni MA, Batista MC, Durao Mde S Jr and Monte JC: Nec-1 protects against nonapoptotic cell death in cisplatin-induced kidney injury. Ren Fail 34: 373-377, 2012.

8. Koudstaal S, Oerlemans MI, Van der Spoel TI, Janssen AW, Hoefer IE, Doevendans PA, Sluijter JP and Chamuleau SA Necrostatin-1 alleviates reperfusion injury following acute myocardial infarction in pigs. Eur J Clin Invest 45: 150-159, 2015

9. Dionisio PE, Oliveira SR, Amaral JS and Rodrigues CM: Loss of microglial parkin inhibits necroptosis and contributes to neuroinflammation. Mol Neurobiol 56: 2990-3004, 2019.

10. Liu ZY, Wu B, Guo YS, Zhou YH, Fu ZG, Xu BQ, Li JH, Jing L, Jiang JL, Tang J and Chen ZN: Necrostatin-1 reduces intestinal inflammation and colitis-associated tumorigenesis in mice. Am J Cancer Res 5: 3174-3185, 2015.

11. Han W, Xie J, Fang Y, Wang Z and Pan H: Nec-1 enhances shikonin-induced apoptosis in leukemia cells by inhibition of RIP-1 and ERK1/2. Int J Mol Sci 13: 7212-7225, 2012.

12. Zhe-Wei S, Li-Sha G and Yue-Chun L: The role of necroptosis in cardiovascular disease. Front Pharmacol 9: 721, 2018.

13. Shan B, Pan H, Najafov A and Yuan J: Necroptosis in development and diseases. Genes Dev 32: 327-340, 2018.

14. Dhuriya YK and Sharma D: Necroptosis: A regulated inflammatory mode of cell death. J Neuroinflammation 15: 199, 2018

15. Linkermann A, Hackl MJ, Kunzendorf U, Walczak H Krautwald S and Jevnikar AM: Necroptosis in immunity and ischemia-reperfusion injury. Am J Transplant 13: 2797-2804, 2013.

16. Wu W, Liu P and Li J: Necroptosis: An emerging form of programmed cell death. Crit Rev Oncol Hematol 82: 249-258, 2012.

17. Dhib-Jalbut S and Kalvakolanu DV: Microglia and necroptosis: The culprits of neuronal cell death in multiple sclerosis Cytokine 76: 583-584, 2015.

18. Ofengeim D, Ito Y, Najafov A, Zhang Y, Shan B, DeWitt JP, Ye J, Zhang X, Chang A, Vakifahmetoglu-Norberg $\mathrm{H}$, et al: Activation of necroptosis in multiple sclerosis. Cell Rep 10: 1836-1849, 2015.

19. Lu C, Chen X, Wang $\mathrm{Q}, \mathrm{Xu} X$ and $\mathrm{Xu} B$ : TNF $\alpha$ promotes glioblastoma A172 cell mitochondrial apoptosis via augmenting mitochondrial fission and repression of MAPK-ERK-YAP signaling pathways. Onco Targets Ther 11: 7213-7227, 2018.

20. Shioda M, Muneta T, Tsuji K, Mizuno M, Komori K, Koga H and Sekiya I: TNF $\alpha$ promotes proliferation of human synovial MSCs while maintaining chondrogenic potential. PLoS One 12: e0177771, 2017.

21. Bryan C, Sammour I, Guerra K, Sharma M, Dapaah-Siakwan F, Huang J, Zambrano R, Benny M, Wu S and Young K: TNFo-stimulated protein 6 (TSG-6) reduces lung inflammation in an experimental model of bronchopulmonary dysplasia. Pediatr Res 85: 390-397, 2019.

22. Lee E, Ouzounova M, Piranlioglu R, Ma MT, Guzel M, Marasco D, Chadli A, Gestwicki JE, Cowell JK, Wicha MS, et al: The pleiotropic effects of TNF $\alpha$ in breast cancer subtypes is regulated by TNFAIP3/A20. Oncogene 38: 469-482, 2019.

23. Pegoretti V, Baron W, Laman JD and Eisel ULM: Selective modulation of TNF-TNFRs signaling: Insights for multiple sclerosis treatment. Front Immunol 9: 925, 2018.

24. Zhou Y, Taylor B, van der Mei I, Stewart N, Charlesworth J, Blizzard L, Ponsonby AL, Dwyer T, Pittas F and Simpson S Jr: Genetic variation in PBMC-produced IFN- $\gamma$ and TNF- $\alpha$ associations with relapse in multiple sclerosis. J Neurol Sci 349: 40-44, 2015.

25. Dendrou CA, Bell JI and Fugger L: A clinical conundrum: The detrimental effect of TNF antagonists in multiple sclerosis Pharmacogenomics 14: 1397-1404, 2013.

26. Haji N, Mandolesi G, Gentile A, Sacchetti L, Fresegna D, Rossi S, Musella A, Sepman H, Motta C, Studer V, et al: TNF- $\alpha$-mediated anxiety in a mouse model of multiple sclerosis. Exp Neurol 237 296-303, 2012.
27. Jurewicz AM, Walczak AK and Selmaj KW: Shedding of TNF receptors in multiple sclerosis patients. Neurology 53: 1409-1414, 1999.

28. Kamali-Sarvestani E, Nikseresht A, Aflaki E, Sarvari J and Gharesi-Fard B: TNF-alpha, TNF-beta and IL-4 gene polymorphisms in Iranian patients with multiple sclerosis. Acta Neurol Scand 115: 161-166, 2007.

29. Wang Y, Bi Y, Xia Z, Shi W, Li B, Li B, Chen L and Guo L: Butylphthalide ameliorates experimental autoimmune encephalomyelitis by suppressing PGAM5-induced necroptosis and inflammation in microglia. Biochem Biophys Res Commun 497: 80-86, 2018.

30. Alwahaibi NY, Alkhatri AS and Kumar JS: Hematoxylin and eosin stain shows a high sensitivity but sub-optimal specificity in demonstrating iron pigment in liver biopsies. Int $\mathrm{J}$ Appl Basic Med Res 5: 169-171, 2015.

31. Chen Y, Balasubramaniyan V, Peng J, Hurlock EC, Tallquist M, $\mathrm{Li} \mathrm{J}$ and Lu QR: Isolation and culture of rat and mouse oligodendrocyte precursor cells. Nat Protoc 2: 1044-1051, 2007.

32. Tao F, Tian X, Lu M and Zhang Z: A novel lncRNA, Lnc-OC1, promotes ovarian cancer cell proliferation and migration by sponging miR-34a and miR-34c. J Genet Genomics 45: 137-145, 2018.

33. Zhang Z, Zhang B, Li W, Fu L, Fu L, Zhu Z and Dong JT: Epigenetic silencing of miR-203 upregulates SNAI2 and contributes to the invasiveness of malignant breast cancer cells. Genes Cancer 2: 782-791, 2011.

34. Livak KJ and Schmittgen TD: Analysis of relative gene expression data using real-time quantitative PCR and the 2(-Delta Delta C(T)) method. Methods 25: 402-408, 2001.

35. Lenhausen AM, Wilkinson AS, Lewis EM, Dailey KM, Scott AJ, Khan S and Wilkinson JC: Apoptosis inducing factor binding protein PGAM5 triggers mitophagic cell death that is inhibited by the ubiquitin ligase activity of X-linked inhibitor of apoptosis. Biochemistry 55: 3285-3302, 2016.

36. Liao L, Shang L, Li N, Wang S, Wang M, Huang Y, Chen D, Huang J and Xiong K: Mixed lineage kinase domain-like protein induces RGC-5 necroptosis following elevated hydrostatic pressure. Acta Biochim Biophys Sin (Shanghai) 49: 879-889, 2017.

37. Cudrici C, Niculescu T, Niculescu F, Shin ML and Rus H: Oligodendrocyte cell death in pathogenesis of multiple sclerosis: Protection of oligodendrocytes from apoptosis by complement. J Rehabil Res Dev 43: 123-132, 2006.

38. Van Kaer L, Postoak JL, Wang C, Yang G and Wu L: Innate, innate-like and adaptive lymphocytes in the pathogenesis of MS and EAE. Cell Mol Immunol 16: 531-539, 2019.

39. Robinson AP, Harp CT, Noronha A and Miller SD: The experimental autoimmune encephalomyelitis (EAE) model of MS: Utility for understanding disease pathophysiology and treatment. Handb Clin Neurol 122: 173-189, 2014.

40. Yuan J,Amin P and Ofengeim D: Necroptosis and RIPK1-mediated neuroinflammation in CNS diseases. Nat Rev Neurosci 20: 19-33, 2019.

41. Shao L, Yu S, Ji W, Li H and Gao Y: The Contribution of Necroptosis in Neurodegenerative Diseases. Neurochem Res 42: 2117-2126, 2017

42. Zhang S, Wang Y, Li D, Wu J, Si W and Wu Y: Necrostatin-1 attenuates inflammatory response and improves cognitive function in chronic ischemic stroke mice. Medicines (Basel) 3: E16, 2016.

43. Chen Y, Zhang L, Yu H, Song K, Shi J, Chen L and Cheng J Necrostatin-1 improves long-term functional recovery through protecting oligodendrocyte precursor cells after transient focal cerebral ischemia in mice. Neuroscience 371: 229-241, 2018.

44. Fan H, Tang HB, Kang J, Shan L, Song H, Zhu K, Wang J, Ju G and Wang YZ: Involvement of endoplasmic reticulum stress in the necroptosis of microglia/macrophages after spinal cord injury. Neuroscience 311: 362-373, 2015. 